\title{
Pathological Fracture of the Femoral Neck due to Tophaceous Gout: An Unusual Case of Gout
}

\author{
Yoo-Sun Jeon, MD, Deuk-Soo Hwang, MD, PhD*, Jung-Mo Hwang, MD, PhD*, \\ Jeong-Kil Lee, MD*, Young-Cheol Park, MD* \\ Department of Orthopaedic Surgery, Barun Mind Hospital, Daejeon, Korea \\ Department of Orthopaedic Surgery, Chungnam National University School of Medicine, Daejeon, Korea*
}

A 48-year-old man visited the emergency room with right hip pain that started abruptly while walking out of the bathroom. Computed tomography showed an intraosseous mass in the femoral neck. The patient had a 15-year history of gout and had numerous bilateral tophi in his hands, feet, knees, and elbows. After operation, we diagnosed a pathological fracture due to intraosseous tophi. Patients with hip pain who have many subcutaneous tophi and long-standing gout should be diagnosed carefully. Peri-hip joint pain caused by gout is uncommon, however, if a patient complains of pain, a simple X-ray may be required. If intraosseous tophi are present, appropriate treatment (e.g., strict hyperuricemia control with or without prophylactic internal fixation), may be required before fracture occurs.

Key Words: Gout arthritis, Femoral neck fracture, Intraosseous tophi, Subcutaneous tophi

Recent data suggest that gout is one of the most common musculoskeletal disorders in the general population, with a prevalence of approximately 8.4 per 1,000 persons of all ages ${ }^{1)}$. Gout involves the deposition of urate crystals in the synovial membranes, cartilage, periarticular tissues, and subcutaneous tissues out of the joints, leading to bone erosion $^{2}$. Bone erosion due to tophi is common, while urate

Submitted: April 25, 2019 1st revision: July 23, 2019

Final acceptance: August 2, 2019

Address reprint request to

Deuk-Soo Hwang, MD, PhD

[https://orcid.org/0000-0003-1009-3784]

Department of Orthopaedic Surgery, Chungnam National

University School of Medicine, 282 Munwha-ro, Jung-gu, Daejeon

35015, Korea

TEL: +82-42-338-2480 FAX: +82-42-338-2482

E-mail: dshwangdenu.ac.kr

This is an Open Access article distributed under the terms of the Creative Commons Attribution Non-Commercial License (http://creativecommons. org/licenses/by-nc/4.0) which permits unrestricted non-commercial use, distribution, and reproduction in any medium, provided the original work is properly cited. crystals form intraosseous tophi more rarely. Intraosseous tophi have been associated with pathological fractures following minor trauma or even in the absence of trauma, with the most common fracture site being the patella ${ }^{3}$.

We encountered a patient with a pathological fracture due to intraosseous tophi of the femoral neck, a rare occurrence with only a few case reports of tophi around the hip joint or pelvic bones ${ }^{4-6}$. It is a rare case of pain caused by tophi around the hip. However, the authors emphasize the need for careful differential diagnosis to prevent pathological fractures in patients with hip pain and long-standing gout and multiple tophi, as in this case.

\section{CASE REPORT}

A 48-year-old man visited the emergency room with right hip pain that started abruptly while walking out of the bathroom. Simple X-rays revealed a fracture line in the femoral neck and an oval, sclerotic marginal cystic mass around the femoral neck. Computed tomography 


\section{Hip \& Pelvis}

Yoo-Sun Jeon et al. Pathological Fracture of the Femoral Neck due to Tophaceous Gout

(CT) revealed an intraosseous mass in the femoral neck (Fig. 1). In addition to the identified mass, CT findings revealed a destructed hip joint due to intra-articular mass. The patient had been suffering from gout for 15 years and had numerous bilateral tophi in his hands, feet, knees, and elbows. Therefore, differential diagnosis of femoral pathological fracture by intraosseous tophi was necessary (Fig. 2). A blood test in the emergency room revealed levels of uric acid $(9.6 \mathrm{mg} / \mathrm{dL})$, C-reactive protein $(0.8 \mathrm{mg} / \mathrm{dL})$, erythrocyte sedimentation rate $(45$ $\mathrm{mm} / \mathrm{hr})$, and white blood cell $\left(6.07 \times 10^{3} / \mu \mathrm{L}\right)$.

This patient had lived without changing lifestyle or dietary control, and over the previous 15 years, had only visited a private hospital when experiencing acute pain. For many years, tophi had developed in both hands and feet. Roughly six months before visiting the emergency
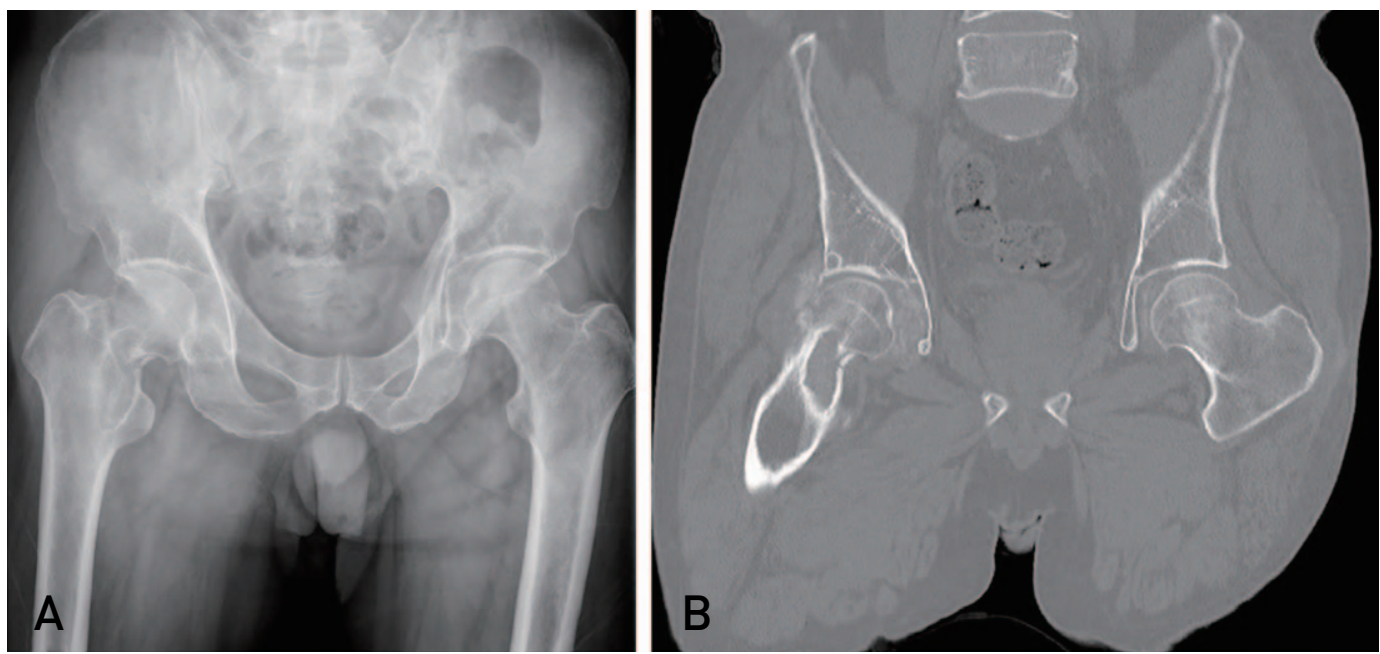

Fig. 1. Initial X-ray and computed tomography (CT) scan at the emergency room. (A) Simple X-ray reveals an oval, sclerotic marginal cystic mass and fracture line around the femoral neck. (B) CT scan reveals an intraosseous mass in the femoral neck.
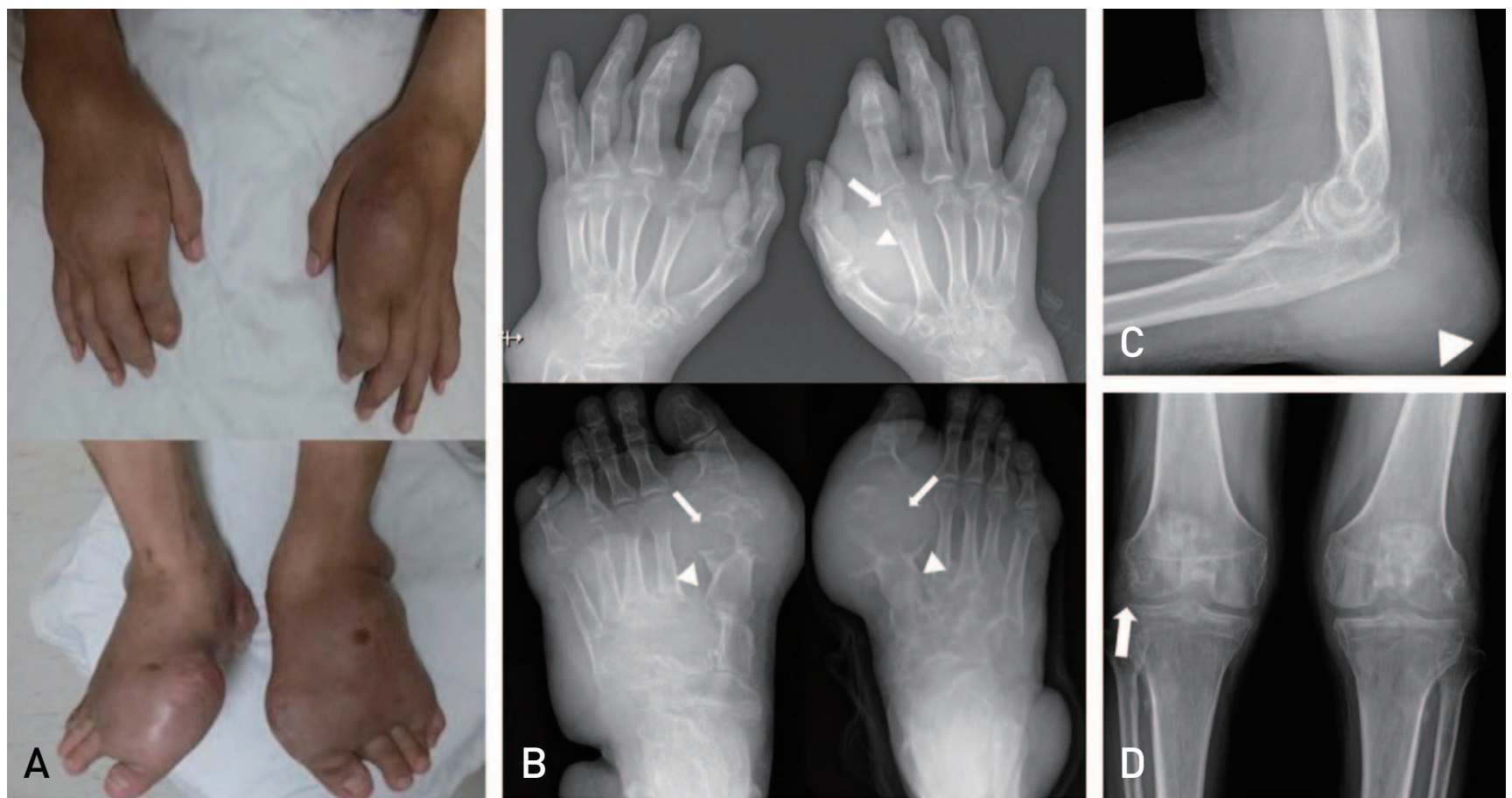

Fig. 2. Gross photos and simple X-rays. (A) Gross photos of hands and feet. The patient has many subcutaneous tophi. (B) Both hands and feet X-rays. Typical 'punched-out lesions (arrows) and overhanging margins (arrowheads) are seen. Elbow (C) and knee (D) X-rays. Arrowhead indicates subcutaneous tophi of left elbow and arrow indicates intra-articular tophi. 


\section{Hip \& Pelvis}

Hip Pelvis 31(4): 238-241, 2019

room, the tophi of both hands and feet increased rapidly, causing tenderness and heat sensation. From that time, he had been prescribed febuxostat $(40 \mathrm{mg}$; qd [quaque die, everyday]), colchicine ( $0.6 \mathrm{mg}$; bid [bis in die, twice a day]), and aceclofenac (100 $\mathrm{mg}$ bid) by the rheumatologist. The serum uric acid level was often 8 to $10 \mathrm{mg} / \mathrm{dL}$. The size of the tophi and serum uric acid level did not decrease after taking the medication.

We proposed screw fixation and curettage with bone graft as a surgical method when the patient was hospitalized. However, this patient wanted a total hip replacement, because it was difficult to manage postoperative care (e.g., walking with crutches), due to the pain in both hands and feet; a total hip replacement was therefore performed. During the operation, a chalky and white paste-like mass were removed from the articular cavity (Fig. 3A). The mass showed negative birefringence on the polarized microscope, and was histologically confirmed as gout (Fig. 3B, C). Postoperatively, the patient was prescribed febuxostat ( $80 \mathrm{mg} \mathrm{qd}$ ) and consumed a low-purine diet for lifestyle correction. There was no pain in either hip joint for two years postoperatively (Fig. 3D), although intermittent pain was noted in both hands and feet. This case was approved by our institutional review board (CNUH 2019-01-075) and informed consent was obtained from patient.

\section{DISCUSSION}

This report presents an unusual aspect of gout involvement in the hip joint. The hip joint involvement of gouty arthritis is very rare, and the pathological femoral neck fracture was first reported in $2006^{4}$. The joints most commonly involved in gouty arthritis are the first metatarsophalangeal joints, followed by the ankles, knees, and tarsal bones, because urate deposition occurs at extremity temperatures lower than $37^{\circ} \mathrm{C}^{6}$. Gout arthritis involvement in atypical areas is usually accompanied by renal failure or chronic gouty arthritis with inadequate treatment. This patient had normal renal function but long-standing uncontrolled gouty arthritis. Consequently, if a patient has pain around the hip and long-standing, uncontrolled gouty arthritis, like
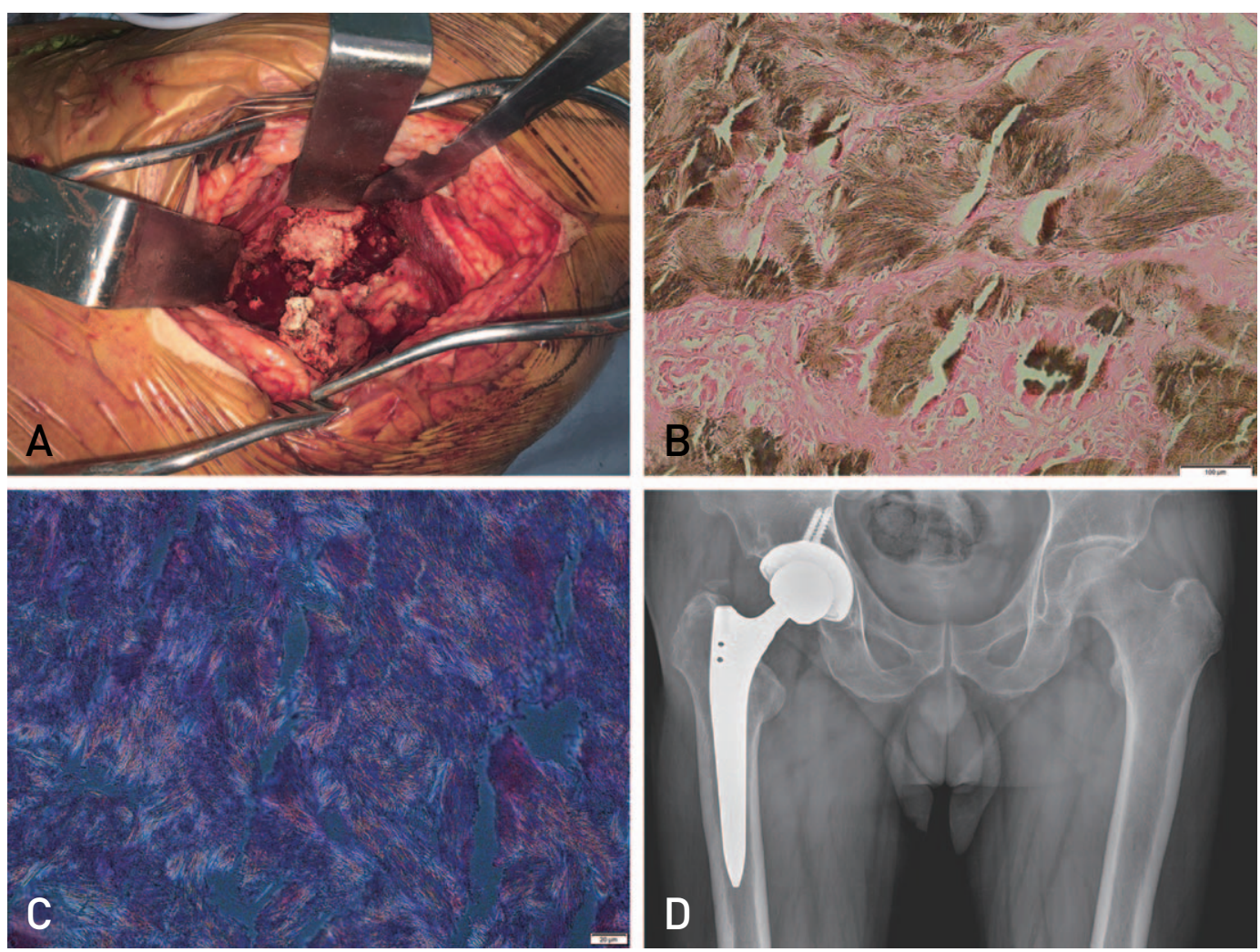

Fig. 3. (A) Gross photo obtained during operation. A chalky and paste-like mass is shown. (B) Photograph of pathology. Fibrotic and necrotic changes are seen around tophi. And only few normal cells are seen (H\&E, $\times 100)$. (C) The mass shows negative birefringence on the polarized microscope. (D) The last follow-up X-ray. There was no pain in either hip joint for two years postoperatively. 
Yoo-Sun Jeon et al. Pathological Fracture of the Femoral Neck due to Tophaceous Gout

this case, or renal failure, physicians should carefully consider that the differential diagnosis may include pathologic fracture by gout. Involvement of the hip joint due to gout is easy to mistake for a hip joint fracture or lumbar herniated intervertebral disc, and the correct diagnosis is likely to be delayed. Before the characteristic radiological findings appear (e.g., punched-out lesions, overhanging margins), simple X-ray findings may be unclear $^{7)}$. CT or magnetic resonance imaging can be used to obtain an accurate diagnosis and dual-energy CT can be helpful. Microscopic analysis of joint fluid using ultrasound-guided aspiration may also be helpful for a definite diagnosis.

Dalbeth et al. ${ }^{8}$ demonstrated a close relationship between bone erosion and the presence and size of intraosseous tophi and suggested that subcutaneous tophi serve as a marker of underlying joint and bone damage in gout. Therefore, physicians should be aware of the possibility of pathological fracture due to intraosseous tophi patients with many subcutaneous tophi. The established operative procedure for large intraosseous tophi is curettage and bone grafting. There is also a report suggesting favorable results using curettage and calcium phosphate cement to fill intraosseous tophi of the talus ${ }^{9}$. If the fracture risk is high, prophylactic internal fixation should be performed with reference to Mirels' criteria.

Zadaka et al. ${ }^{10)}$ emphasized that acute arthritis should be distinguished from septic arthritis and crystal disease in patients undergoing arthroplasty to avoid unnecessary operative procedures. It is necessary to include gout in the differential diagnosis of acute arthritis in arthroplasty joints in patients with a history of long-standing gout or underlying disease (e.g., renal dysfunction). Ultrasound can be useful for evaluating hip joint pain after arthroplasty, for aspiration without nerve or vascular damage, and to visualize intra-articular tophi.

We conclude that patients with hip pain who have many subcutaneous tophi and long-standing gout should be diagnosed carefully. Peri-hip joint pain caused by gout is uncommon, however, if the patient complains of pain, a simple X-ray may be needed. If intraosseous tophi are present, appropriate treatment (e.g., strict hyperuricemia control with or without prophylactic internal fixation), may be required before fracture occurs.

\section{CONFLICT OF INTEREST}

The authors declare that there is no potential conflict of interest relevant to this article.

\section{REFERENCES}

1.Lawrence RC, Helmick CG, Arnett FC, et al. Estimates of the prevalence of arthritis and selected musculoskeletal disorders in the United States. Arthritis Rheum. 1998;41: 778-99.

2. Agudelo CA, Wise CM. Gout: diagnosis, pathogenesis, and clinical manifestations. Curr Opin Rheumatol. 2001;13: 234-9.

3. Nguyen C, Ea HK, Palazzo E, Lioté F. Tophaceous gout: an unusual cause of multiple fractures. Scand J Rheumatol. 2010;39:93-6.

4. Bates TA, Renner JB, Jonas BL. Pathologic fracture of the hip due to severe gouty arthritis. J Rheumatol. 2006;33:1889-90.

5. Burnham J, Fraker K, Steinbach H. Pathologic fractures in an unusual case of gout. AJR Am J Roentgenol. 1977;129: 1116-9.

6. Loeb JN. The influence of temperature on the solubility of monosodium urate. Arthritis Rheum. 1972;15:189-92.

7. Mantle B, Gross P, Lopez-Ben R, Alarcón GS. Hip pain as the presenting manifestation of acute gouty sacroiliitis. $J$ Clin Rheumatol. 2001;7:112-4.

8. Dalbeth N, Clark B, Gregory K, et al. Mechanisms of bone erosion in gout: a quantitative analysis using plain radiography and computed tomography. Ann Rheum Dis. 2009;68:1290-5.

9. Morino T, Fujita M, Kariyama K, Yamakawa H, Ogata T, Yamamoto H. Intraosseous gouty tophus of the talus, treated by total curettage and calcium phosphate cement filling: a case report. Foot Ankle Int. 2007;28:126-8.

10.Zadaka A, Gioe T, Gertner E. Acute crystal-induced arthritis following arthroplasty. J Knee Surg. 2010;23:17-20. 